\title{
Endobronchial Manifestation of Methotrexate-induced Lymphoproliferative Disorder
}

Tatsuyoshi Ikeue, Hiroaki Kawachi, Hiroshi Yoshida, Eiichiro Tanaka, Susumu Noguchi, Akari Fukao, Satoshi Terashita, Tomoko Tajiri, Sadao Horikawa and Takakazu Sugita

\begin{abstract}
:
Lymphoproliferative disorders can occur in patients with autoimmune disorders who undergo long-term methotrexate therapy (MTX-LPD). Although the manifestations of MTX-LPD are diverse, little attention is paid to endobronchial involvement. We herein describe two patients with MTX-LPD who presented with parenchymal pulmonary tumors and endobronchial involvement of LPD; one had lymphomatoid gramulomatosis and the other LPD. The patients had no tumors adjacent to the endobronchial lesions. The endobronchial findings included multiple protruded mucosal lesions covered with white material, which was pathologically consistent with LPD. Recognition of the findings may help in making an earlier diagnosis of MTX-LPD in appropriate settings.
\end{abstract}

Key words: methotrexate-induced lymphoproliferative disorder, endobronchial involvement, lymphomatoid granulomatosis

(Intern Med 58: 1597-1603, 2019)

(DOI: 10.2169/internalmedicine.2109-18)

\section{Introduction}

Lymphoproliferative disorders (LPDs) associated with iatrogenic immunodeficiency involve various types of lymphoid and plasmacytic proliferations that mainly occur after transplantation (post-transplant lymphoproliferative disorders) (1); however, a small number of cases occur in patients with autoimmune disorders, mainly rheumatoid arthritis (RA), who are treated with long-term low-dose methotrexate therapy (MTX-induced LPD, MTXLPD) $(2,3)$. Many - but not all - LPDs are associated with Epstein-Barr virus (EBV). Morphologically, cases most commonly meet the criteria for diffuse large B cell lymphoma (DLBCL), and less commonly Hodgkin's disease, follicular lymphoma and other types of LPD (4). Practically, the recognition of MTX-LPD is important, since 40\%-70\% of these cases respond to the withdrawal of MTX therapy (although relapse may occur) $(5,6)$.

MTX-LPD may involve various organs, including the lymph nodes, lung, liver, brain and skin; however, little is known about endobronchial involvement. We herein report two MTX-LPD patients on long-term MTX treatment due to RA, which had regressed after the discontinuation of MTX. Notably, this report features findings of the endobronchial manifestations of MTX-LPDs observed by fiberoptic bronchoscopy, which may help in the diagnosis of MTX-LPD when the findings are combined with lung nodules in patients undergoing long-term MTX therapy.

\section{Case Reports}

\section{Patient 1}

A 64-year-old woman who presented with a dry cough that had persisted for 1 month was referred to our hospital to undergo an evaluation for a pulmonary tumor. The patient had a 20-year history of rheumatoid arthritis and had received prednisone ( $2 \mathrm{mg}$, daily) and MTX ( $8 \mathrm{mg}$, weekly) for more than 9 years (cumulative dose of MTX: $>3,744$ $\mathrm{mg}$ ). She had never smoked or consumed alcohol.

Her vital signs on presentation were as follows: body temperature, $35.9^{\circ} \mathrm{C}$; pulse rate, 79 beats per minute; blood pressure, $118 / 74 \mathrm{mmHg}$ and respiratory rate, 18 breaths per 

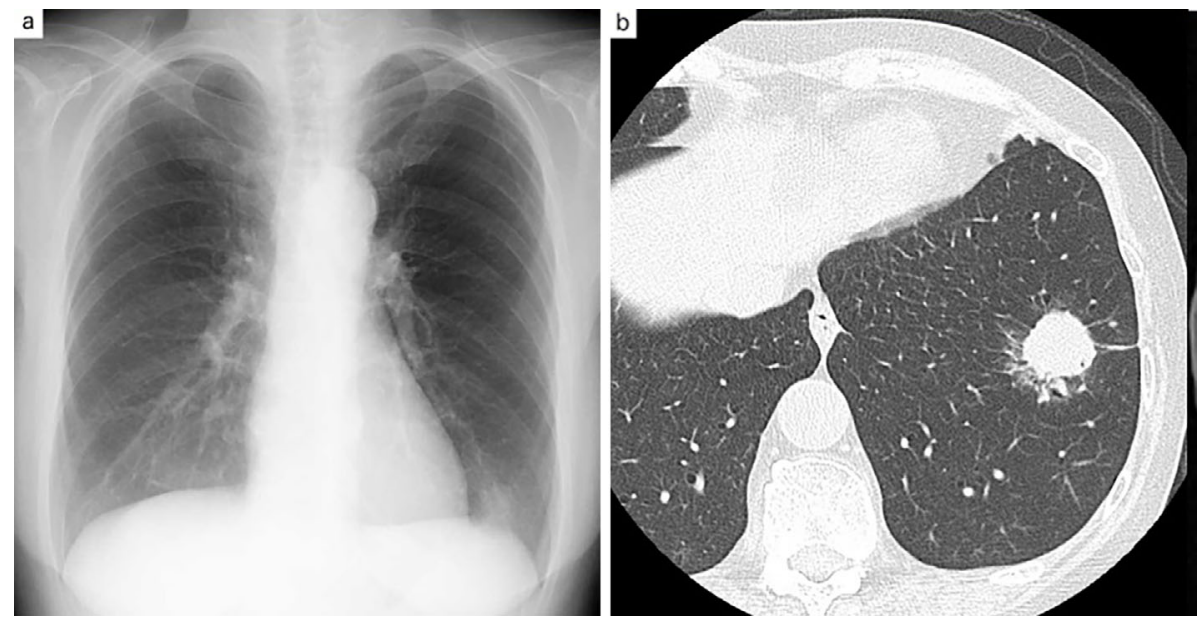

Figure 1. Chest X-ray and computed tomography (CT) at the presentation of Patient 1. a: Chest $\mathrm{X}$-ray shows a tumor shadow in the left lower field. b: Computed tomography (CT) shows a poorly defined tumor $\mathbf{2} \mathrm{cm}$ in diameter with a shaggy margin and pleural indentation in the lower lobe of the left lung.
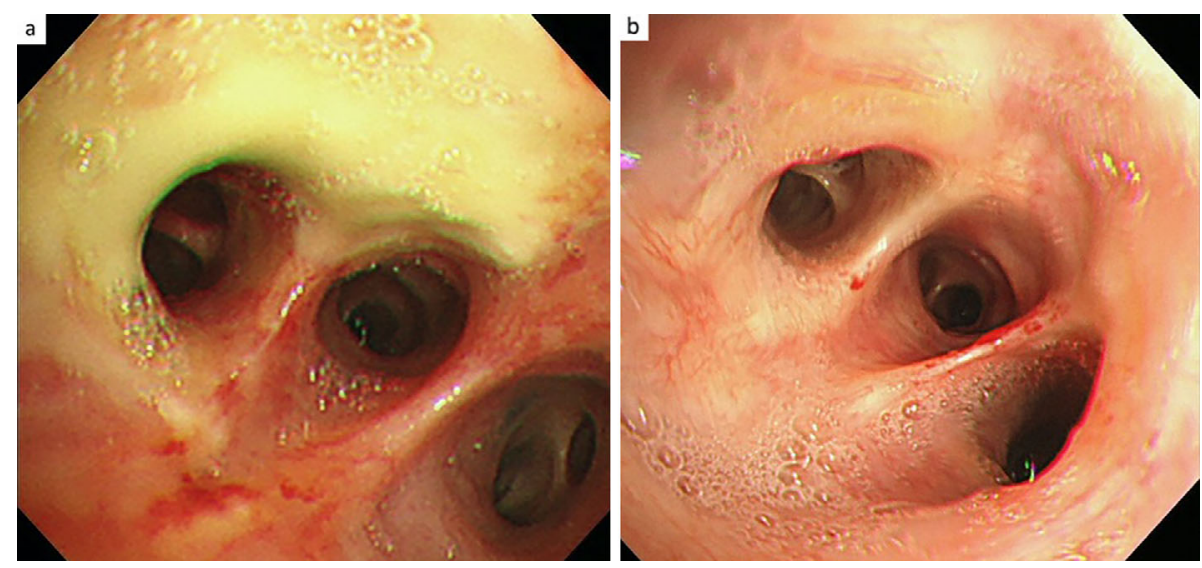

Figure 2. a: A fiberoptic bronchoscopic image at the basal bronchus of Patient 1 shows slightly protruded mucosal lesions covered with white material. b: A fiberoptic bronchoscopic image after the withdrawal of MTX. The endobronchial lesions are resolved.

minute. Auscultation of breath sounds was normal. Physical examinations revealed no palpable superficial lymph nodes, or neurological or dermatological abnormalities. Laboratory tests showed slightly elevated C-reactive protein (CRP) $(0.56$ $\mathrm{mg} / \mathrm{dL})$, lactate dehydrogenase (LD) (216 UI/dL) and interleukin-2 receptor (IL-2R) (797 U/mL) levels. The levels of tumor markers, including carcinoembrionic antigen (CEA), cytokeratin-19 fragment (CYFRA), neuron-specific enolase (NSE) and pro-gastrin-releasing peptide (Pro-GRP), were all within the normal ranges. The patient's beta-D glucan, Aspergillus antigen and specific IgE antibody for Aspergillus titers were also normal.

Chest X-ray showed a nodular shadow in the lower field of the left lung (Fig. 1a). Chest computed tomography (CT) showed a tumor shadow $2 \mathrm{~cm}$ in diameter with a shaggy margin and pleural indentation in the lower lobe of the left lung along with multiple smaller nodules in the right lung (Fig. 1b). Brain magnetic resonance imaging (MRI) showed multiple ring-enhanced intracranial tumors with peritumoral edema. Abdominal CT showed no abnormalities. Fiberoptic bronchoscopy demonstrated slightly protruded mucosal lesions covered with white material in the right basal bronchus and the orifices of the right B8 and B9 bronchi (Fig. 2a). A transbronchial biopsy of the left pulmonary nodule did not yield sufficient material for a definite diagnosis. A biopsy of the right bronchial lesions showed granulomatous aggregation of lymphocytes (Fig. 3a); however, the results of Periodic acid-Schiff (PAS), Grocott and ZiehlNeelsen staining were all negative, and bronchial wash cultures grew no causative organisms. Thus, the patient underwent video-assisted thoracosurgery (VATS) to resect the left lower lobe tumor.

The histopathology of the lung tumor demonstrated widespread necrosis surrounded by lymphocyte and macrophage infiltration; however, there were no specific findings (e.g., atypical cells, granulomas or pathogens) (Fig. 3d). Repeated 


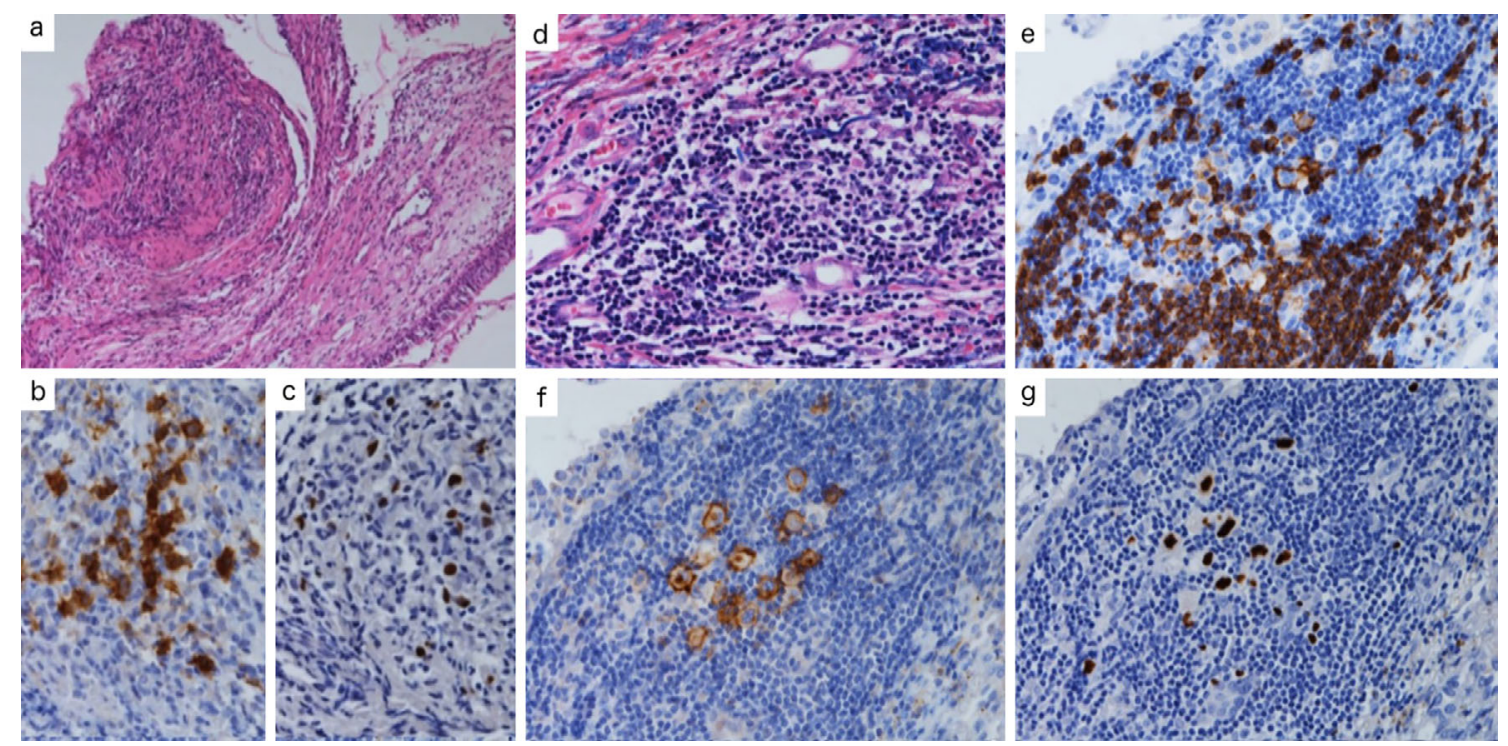

Figure 3. A histological examination of the transbronchial biopsy (a-c) and the lung tumor (d-g) of Patient 1. a: An aggregate of lymphocytes is seen in the bronchial submucosa [Hematoxylin and Eosin (H\&E) staining, $\times 100]$. Immunohistochemical staining of the large atypical lymphocytes revealed CD20 (b: $\times 400)$ and EBV-encoded small RNA in situ hybridization (EBER-ISH) (c: $\times 400)$ positivity. d: An aggregate of the large atypical lymphocytes is seen surrounding widespread necrosis (H\&E staining, $\times 400)$. Immunohistochemical staining of the large atypical lymphocytes revealed CD20 (e: $\times 400)$, CD30 (f: $\times 400)$ and EBER-ISH $(g: \times 400)$ positivity.

brain MRI showed that the brain tumors were increasing in size; thus, the patient underwent surgical resection of the larger brain tumor of the left hemisphere. The pathological examination demonstrated widespread necrosis surrounded by lymphocyte infiltration in the angiocentric distribution (Fig. 4a). The lymphoid infiltrate contained large atypical lymphocytes that were positive for $\mathrm{CD} 20$ and CD30 (Fig. 4b, c). EBV-encoded small RNA in situ hybridization (EBER-ISH) was positive ( $>50$ cells/high-power field) (Fig. 4d). These findings were consistent with lymphomatoid granulomatosis (grade 3). A history of long-term low-dose MTX treatment warranted a diagnosis of MTX-LPD. A reevaluation of the transbronchial biopsy and VATS specimens revealed that they were positive for $\mathrm{CD} 20, \mathrm{CD} 30$ and EBER-ISH (Fig. 3b, c, e-g).

One month after the discontinuation of MTX, the residual brain tumors and peritumoral edema regressed almost completely. A month later, the endobronchial findings returned to normal (Fig. 2b), and the residual lung tumors shrank in size, with the exception of a new small nodule that emerged in the lower lobe of the right lung. The size of this nodule was unchanged 12 months later.

\section{Patient 2}

A 42-year-old man presented to our hospital with a high fever and cough. He had received prednisone (10 mg, daily) and MTX (7.5-12.5 mg, weekly) for 10 years (cumulative dose: $3,975 \mathrm{mg}$ ) for the treatment of RA and polyangitis nodosa. He had a 17-pack-year smoking history (he had quit smoking 5 years before presentation) and had never con- sumed alcohol.

His vital signs were as follows: body temperature, $38.8^{\circ} \mathrm{C}$; pulse rate, 128 beats per minute; blood pressure, 121/78 $\mathrm{mmHg}$ and oxygen saturation, 98\% (on ambient air). Auscultation of breath sounds was normal. Physical examinations revealed necrotic gingivitis, and the bilateral inguinal lymph nodes were palpable. Laboratory tests showed slightly elevated CRP (7.51 mg/dL), LD (253 UI/dL), CYFRA $(3.17 \mathrm{ng} / \mathrm{mL})$ and IL-2R $(801 \mathrm{U} / \mathrm{mL})$ levels. The levels of other tumor markers, including CEA, NSE and Pro-GRP, were normal. The patient's beta-D glucan level was normal, and his cryptococcal antigen titer was negative.

Chest X-ray revealed a tumor shadow on the left hilum (Fig. 5a). Body computed tomography (CT) showed a tumor of the left hilum with multiple bilateral pulmonary nodules and enlargement of the sublingual and inguinal lymph nodes; however, no mediastinal lymph nodes were enlarged (Fig. 5b). A bronchoscopic examination showed multiple protruded mucosal lesions covered with white material through the trachea, bilateral main and lobar bronchi (Fig. 6a). A pathological study of the biopsy specimens from the inguinal lymph nodes demonstrated extensive necrosis surrounded by lymphocytes (Fig. 7a). An immunohistopathological examination demonstrated that the lymphocytes were positive for CD20 and CD30 (Fig. 7b, c). Immunostaining for latent membrane protein 1 (LMP1) was positive (Fig. 7d), while EBER-ISH was negative. The pathological findings of the transbronchial biopsy specimen were consistent with those of the inguinal lymph nodes (Fig. 7e, f). All of these results and his medical history indi- 

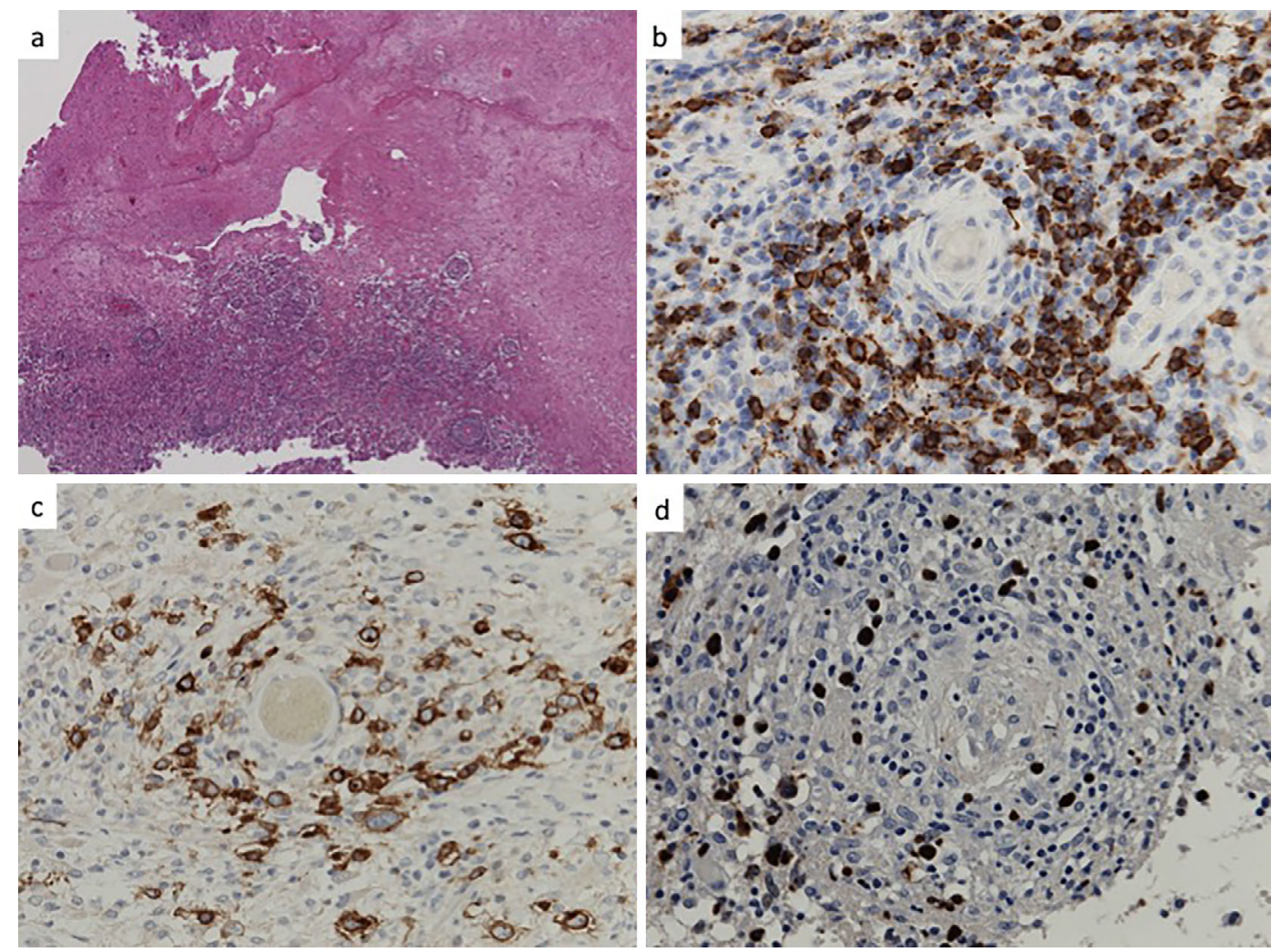

Figure 4. A histological examination of the brain tumor of Patient 1. a: Widespread necrosis surrounded by infiltration of lymphocytes in the angiocentric distribution (Hematoxylin and Eosin staining, $\times 40$ ). Immunohistochemical staining of the large atypical lymphocytes revealed CD20 (b: $\times 400)$, CD30 (c: $\times 400)$ and EBER-ISH (d: $\times 400)$ positivity.
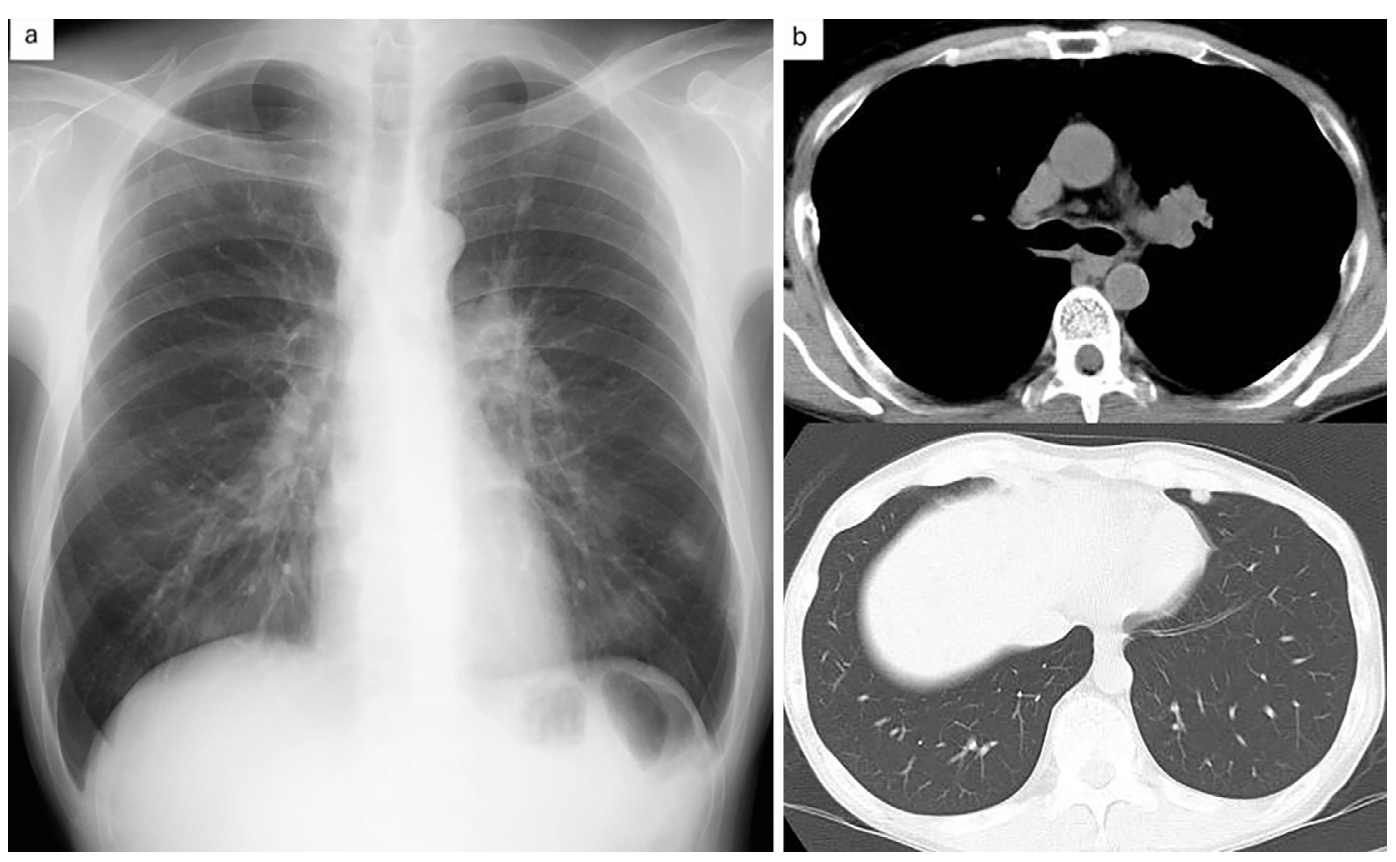

Figure 5. Chest X-ray and computed tomography (CT) at the presentation of Patient 2. a: Chest $X$-ray shows enlargement of the left hilum. b: Chest CT shows an enlarged left hilar tumor (upper) and a small lung nodule in the left lower lobe (lower).

cated a diagnosis of MTX-LPD. The withdrawal of MTX resulted in the prompt regression of all the lesions, including the lesion of the bronchial mucosa (Fig. 6b). 


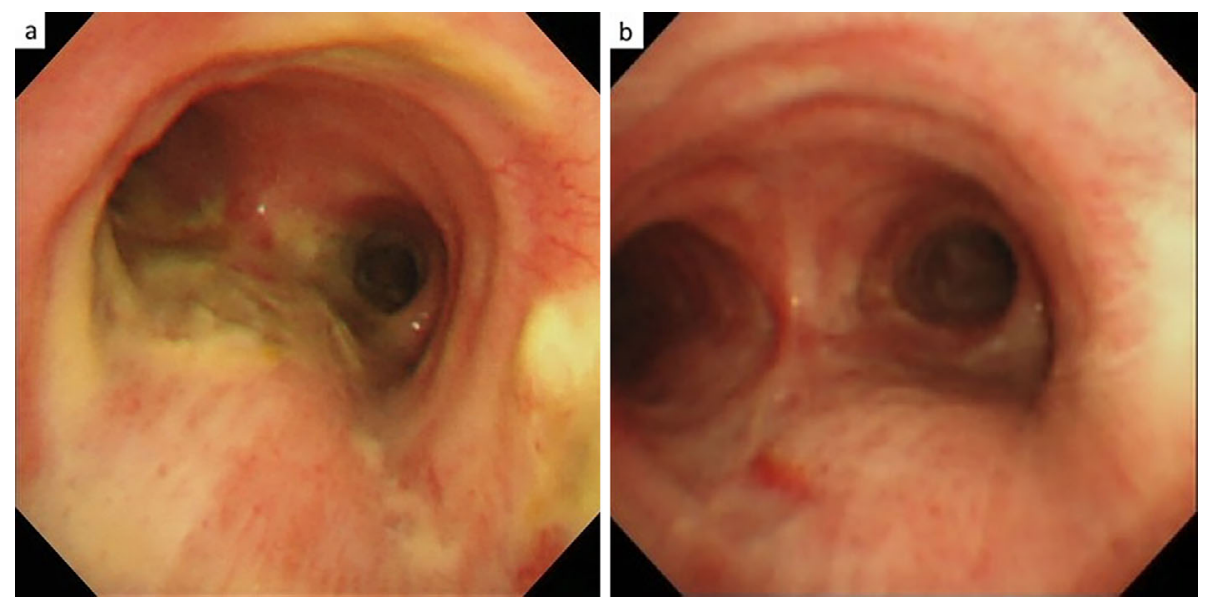

Figure 6. a: A fiberoptic bronchoscopic image at the bifurcation of the trachea of Patient 2 shows multiple slightly protruded tracheobronchial mucosal lesions covered with white material. b: A fiberoptic bronchoscopic image after the withdrawal of MTX. The endobronchial lesions are resolved.
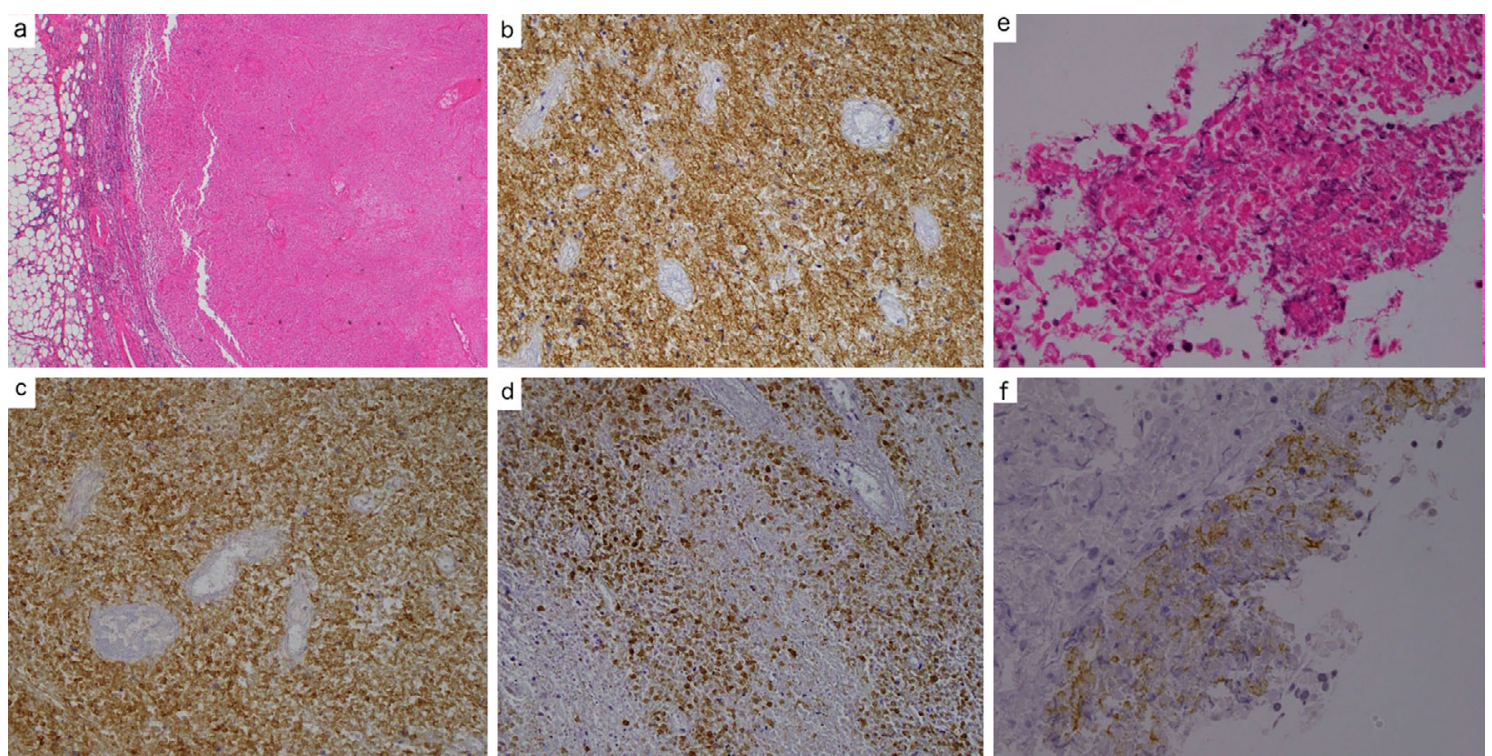

Figure 7. A histological examination of the right inguinal lymph node biopsy (a-d) and the transbronchial biopsy (e, f) specimen of Patient 2. a: Widespread necrosis surrounded by infiltration of lymphocytes [Hematoxylin and Eosin $(H \& E)$ staining, $\times 20]$. Immunohistochemical staining of the atypical lymphocytes revealed CD20 (b: $\times 200), \operatorname{CD30}(c: \times 200)$ and latent membrane protein 1 $($ LMP1) (d: $\times 200)$ positivity. e: A histological examination of the transbronchial biopsy demonstrated necrosis with a small number of lymphocytes (H\&E staining, $\times 400)$. f: Immunohistochemical staining of the lymphocytes revealed CD20 positivity $(\times 400)$.

\section{Discussion}

These two patients with MTX-LPD had multiple endobronchial lesions that were slightly protruded and covered with white material. CT of the chest detected no tumors or enlarged lymph nodes adjacent to the lesions. The histological findings of the biopsy specimens obtained from the endobronchial lesions were consistent with those from the other biopsy sites, and all of the endobronchial lesions regressed after discontinuation of MTX along with the resolution of the tumors at other sites, which indicated that these lesions represented the bronchial mucosal involvement of MTX-LPD rather than the invasion of an extrinsic tumor. Thus far, four case reports have been published describing endobronchial lesions observed in patients with MTXLPD (7-10) (Cases 1-4, in Table). Three cases had direct invasion of the adjacent lymph nodes (Case 1,3) $(7,9)$ or a parenchymal tumor (Case 2) (8) with bronchial stenosis, whereas Case 4 (10) had multiple endobronchial lesions without adjacent extrinsic tumors, which was in line with the findings in our patients.

Lymphomatous involvement of the airways has been recognized infrequently, and two patterns of disease have been 
Table. Reported Cases of Methotrexate-induced Lymphoproliferative Disorders with Endobronchial Involvement.

\begin{tabular}{|c|c|c|c|c|c|c|c|c|}
\hline Case & Age/sex & Histology & Endobronchial findings & $\begin{array}{l}\text { White } \\
\text { material }\end{array}$ & Distribution & $\begin{array}{l}\text { Adjacent } \\
\text { mass }\end{array}$ & $\begin{array}{l}\text { Mediastinal/ } \\
\text { hilar LN }\end{array}$ & Reference \\
\hline 1 & 76/femele & LYG & airway stenosis & - & adjacent mass area & + & $-/+$ & 7 \\
\hline 2 & 69/female & DLBCL & airway obstruction & + & adjacent mass area & + & $-/+$ & 8 \\
\hline 3 & $72 /$ male & PTCL & edema with telangiectasia & - & adjacent mass area & + & $+/+$ & 9 \\
\hline 4 & 73/female & DLBCL & protruding mucosal lesions & + & multiple, diffuse & - & $-/-$ & 10 \\
\hline 5 & 64/female & LYG & protruding mucosal lesions & + & multiple, loalized & - & $-/-$ & Patient 1 \\
\hline 6 & 42/male & LPD & protruding mucosal lesions & + & multiple, diffuse & - & $-/+$ & Patient 2 \\
\hline
\end{tabular}

LYG: lymphomatoid granulomatosis, DLBCL: diffuse large B cell lymphoma, LPD: lymphoproliferative disorder, PTCL: peripheral T cell lymphoma

described for endobronchial non-Hodgkin lymphoma (NHL) (11). Type 1 is characterized by the presence of diffuse submucosal nodules lining bronchoscopically visualized airways in individuals with clinically apparent systemic lymphoma. In this type, pulmonary parenchymal involvement with NHL is dominant. Type 2 is characterized by a localized endobronchial mass of NHL associated with airway obstruction and varying degrees of atelectasis due to extension from the adjacent enlarged lymph nodes. These findings are also typical of Hodgkin's disease (12). Among these six patients with MTX-LPD, the endobronchial findings in Cases 1-3 correspond to Type 2, while those in the remaining three patients, including our two (Cases 4-6), were analogous to Type 1. However, compared with the reports on endobronchial lymphoma, covering with white material was a notable feature of the endobronchial findings in four of the six MTX-LPD cases, especially the three patients who corresponded to Type 1 . These lesions mimic some infectious processes, such as endobronchial tuberculosis and invasive tracheobronchial aspergillosis. In our cases, bronchial lavage and biopsy specimens demonstrated no organisms, including mycobacteria and fungi. Endobronchial findings that show a protruded bronchial mucosa covered with white material associated with pulmonary nodules in RA patients treated with low-dose MTX strongly suggest a diagnosis of MTX-LPD when infections such as tuberculosis and aspergillosis are excluded and warrant an immediate immunohistopathological investigation.

The histological study of the biopsied and resected specimens of Patient 1 showed findings consistent with LYG. LYG is an angiocentric and angiodestructive LPD involving extranodal sites, composed of EBV-positive B cells admixed with reactive $\mathrm{T}$ cells, and may develop as MTX-LPD. LYG predominantly involves the lung (>90\% of cases); other common sites of involvement include the brain, liver and skin, which are seen in approximately one third of the population (13). The presentation may mimic lung cancer with metastasis; thus, the pathological confirmation is crucial. The clinical data on MTX-induced LYG are limited: thus far, there are sporadic case reports of 12 patients (including our Patient 1) with MTX-induced LYG (7, 14-23). Ten of these patients were women, and the median age was 71 (range 65-76). The sites of involvement were as follows: lung, $\mathrm{n}=11$; liver, $\mathrm{n}=5$; central nervous system, $\mathrm{n}=3$; spleen, $\mathrm{n}=2$; bronchial mucosa, $\mathrm{n}=2$; pleural effusion, $\mathrm{n}=2$; and stomach, adrenal gland, lymph node, and nasal septum, $\mathrm{n}=1$ (each). The frequency is consistent with that in the original report by Katzenstein (13). We initially suspected primary lung cancer with brain and contralateral lung metastases and therefore repeatedly performed biopsies. Given that the lung and brain are common sites of LYG involvement, MTXinduced LYG should have been suspected in the differential diagnosis in the setting of long-term MTX therapy for the treatment of RA, despite the rarity of this condition.

Interestingly, approximately $40-70 \%$ of patients with MTX-LPD regress after MTX withdrawal $(5,6)$. The two patients described in the present study showed regression at one month after discontinuation of MTX. However, the CT scans of Patient 1 showed that a small pulmonary nodule had emerged two months after the discontinuation of MTX treatment. One prospective study including 25 MTX-LPD patients from France reported that, among 8 patients who were treated by the withdrawal of MTX alone, 3 achieved remission; however, 2 of these patients relapsed (24). In a Japanese study (25), among 43 of 62 participants who achieved remission after the withdrawal of MTX, 14 (33\%) relapsed. The median duration from MTX withdrawal to relapse was 10.6 (range 0.7 to 35.6 ) months. Patients with Hodgkin's disease and follicular lymphoma showed high relapse rates; however, no information about the relapse rate of patients with LYG was shown because no LYG cases were included in the series. Eight (67\%) of the 12 patients with MTX-LPD mentioned above regressed with the withdrawal of MTX alone, and no cases of recurrence were reported. However, 3 patients $(25 \%)$ died from the progression of LYG, while the mortality rate of DLBCL - a more aggressive type of LPD - was reported to be $38 \%$ in the MTXLPD cohort of the study by Tokuhira et al. (25). Although the new nodule seen in Patient 1 might not have been a recurrent tumor, as it remained unchanged in size 12 months later, continued vigilance is necessary.

In conclusion, endobronchial findings showing multiple protruding mucosal lesions covered with a white material may hasten the diagnosis of MTX-LPD in appropriate settings. More reports should be accumulated to determine whether or not endobronchial involvement of MTX-LPD is more frequent than previously considered and whether the endobronchial findings might contribute to a diagnosis of 


\section{MTX-LPD}

The authors state that they have no Conflict of Interest (COI).

\section{References}

1. Gaulard P, Swerdlow SH, Harris NL, Jaffe ES, Sundström C. Other iatrogenic immunodeficiency-associated lymphoproliferative disorders. In: WHO Classification of Tumours of Haematopoietic and Lymphoid Tissues. 4th ed. Swerdlow SH, Campo E, Harris NL, et al., Eds. IARC, Lyon, 2008: 350-351.

2. Kamel OW, van de Rijn M, Weiss LM, et al. Brief report: reversible lymphomas associated with Epstein-Barr virus occurring during methotrexate therapy for rheumatoid arthritis and dermatomyositis. N Engl J Med 328: 1317-1321, 1993.

3. Hoshida Y, Tomita Y, Zhiming D, et al. Lymphoproliferative disorders in autoimmune diseases in Japan: analysis of clinicopathological features and Epstein-Barr virus infection. Int $\mathrm{J}$ Cancer 108: 443-449, 2004.

4. Hasserjian RP, Chen S, Perkins SL, et al. Immunomodulator agent-related lymphoproliferative disorders. Mod Pathol 22: 15321540, 2009

5. Miyazaki T, Fujimaki K, Shirasugi Y, et al. Remission of lymphoma after withdrawal of methotrexate in rheumatoid arthritis: relationship with type of latent Epstein-Barr virus infection. Am J Hematol 82: 1106-1109, 2007.

6. Tokuhira M, Watanabe R, Nemoto $T$, et al. Clinicopathological analyses in patients with other iatrogenic immunodeficiencyassociated lymphoproliferative diseases and rheumatoid arthritis. Leuk Lymphoma 53: 616-623, 2012.

7. Ochi $\mathrm{N}$, Yamane $\mathrm{H}$, Yamagishi $\mathrm{T}$, Monobe $\mathrm{Y}$, Takigawa $\mathrm{N}$. Methotrexate-induced lymphoproliferative disease: Epstein-Barr virus-associated lymphomatoid granulomatosis. J Clin Oncol 31: e348-e350, 2013

8. Hashimoto A, Matsushita R, Iizuka N, et al. Two patients with rheumatoid arthritis receiving methotrexate therapy who developed malignant lymphoma. Shinyaku To Rinsho (J New Rem Clin) 55: 1986-1903, 2006 (in Japanese).

9. Kamiya Y, Toyoshima M, Suda T. Endobronchial Involvement in Methotrexate-associated Lymphoproliferative Disease. Am J Respir Crit Care Med 193: 1304-1306, 2016.

10. Torii R, Noguchi S, Shimabukuro I, Yoshii C, Yatera K. A case of methotrexate-associated lymphoproliferative disorder with multiple nodules in the lung and bronchus. Kikanshigaku (J Jpn Soc Respir Endosc) 39: 430-435, 2017 (in Japanese).

11. Rose RM, Grigas D, Strattemeir E, Harris NL, Linggood RM. Endobronchial involvement with non-Hodgkin's lymphoma. A clinical-radiologic analysis. Cancer 57: 1750-1755, 1986.

12. Kiani B, Magro CM, Ross P. Endobronchial presentation of Hodgkin lymphoma: a review of the literature. Ann Thorac Surg 76: 967-972, 2003.

13. Katzenstein AL, Carrington $\mathrm{CB}$, Liebow AA. Lymphomatoid granulomatosis: a clinicopathologic study of 152 cases. Cancer $\mathbf{4 3}$ : 360-373, 1979 .

14. Ogata T, Shibata S, Matsuoka E, Nakayama T, Yoshida J, Unoura K. A case of methotrexate-associated lymphomatoid granulomatosis. Haigan (Jpn J Lung Cancer) 53: 234-239, 2013 (in Japanese).

15. Inaba $M$, Ushijim $S$, Hirata $N$, Saisyoji $T$, Kitaoka M, Yoshinaga T. Methotrexate-related lymphomatoid granulomatosis in a patient with rheumatoid arthritis. Nihon Kokyuki Gakkai Zasshi (J Jpn Respir Soc) 49: 597-601, 2011 (in Japnese).

16. Ishihara $\mathrm{S}$, Izumi $\mathrm{H}$, Nagase $\mathrm{D}$, et al. Multiple lung tumors assumed to be rapidly advancing methotrexate-associated lymphoproliferative disease. Shinyaku To Rinsho (J New Rem Clin) 64: 183-189, 2015 (in Japanese).

17. Oiwa H, Mihara K, Kan T, et al. Grade 3 lymphomatoid granulomatosis in a patient receiving methotrexate therapy for rheumatoid arthritis. Intern Med 53: 1873-1875, 2014.

18. Blanchart K, Paciencia M, Seguin A, et al. Fatal pulmonary lymphomatoid granulomatosis in a patient taking methotrexate for rheumatoid arthritis. Minerva Anestesiol 80: 119-120, 2014.

19. Yamakawa T, Kurosawa M, Yonezumi M, Suzuki S, Suzuki H. Methotrexate-related lymphomatoid granulomatosis successfully treated with discontinuation of methotrexate and radiotherapy to brain. Rinsho Ketsueki (Jpn J Clin Hematol) 5: 321-326, 2014 (in Japanese).

20. Kameda H, Okuyama A, Tamaru J, Itoyama S, Iizuka A, Takeuchi T. Lymphomatoid granulomatosis and diffuse alveolar damage associated with methotrexate therapy in a patient with rheumatoid arthritis. Clin Rheumatol 26: 1585-1589, 2007.

21. Schalk E, Krogel C, Scheinpflug K, Mohren M. Lymphomatoid granulomatosis in a patient with rheumatoid arthritis receiving methotrexate: successful treatment with the anti-CD20 antibody mabthera. Onkologie 32: 440-441, 2009.

22. Ito S, Shiraishi M, Iwai Y, et al. A case of methotrexate-associated lymphomatoid granulomatosis diagnosed on autopsy. Jichi Med Univ J 38: 65-69, 2015 (in Japanese, Abstract in English).

23. Shimada K, Matsui T, Kawakami M, et al. Methotrexate-related lymphomatoid granulomatosis: a case report of spontaneous regression of large tumours in multiple organs after cessation of methotrexate therapy in rheumatoid arthritis. Scand J Rheumatol 36: 64-67, 2007.

24. Mariette X, Cazals-Hatem D, Warszawki J, Liote F, Balandraud N, Sibilia J; Investigators of the Club Rhumatismes et Inflammation. Lymphomas in rheumatoid arthritis patients treated with methotrexate: a 3-year prospective study in France. Blood 99: 3909-3915, 2002.

25. Tokuhira M, Saito S, Okuyama A, et al. Clinicopathologic investigation of methotrexate-induced lymphoproliferative disorders, with a focus on regression. Leuk Lymphoma 59: 1143-1152, 2018.

The Internal Medicine is an Open Access journal distributed under the Creative Commons Attribution-NonCommercial-NoDerivatives 4.0 International License. To view the details of this license, please visit (https://creativecommons.org/licenses/ by-nc-nd/4.0/).

(C) 2019 The Japanese Society of Internal Medicine Intern Med 58: 1597-1603, 2019 